$$
\begin{aligned}
& \text { Pontifícia Universidade } \text { Católica }_{\text {a }} \\
& \text { DO RIO DE JANEIRO }
\end{aligned}
$$

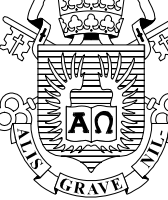

Jorge Luiz Correia de Araújo

\title{
A Criação de Valor Influenciando o Posicionamento Estratégico - Estudo de Caso do Grupo RCH
}

\section{Dissertação de Mestrado}

Dissertação apresentada como requisito parcial para obtenção do grau de Mestre pelo Programa de Pósgraduação em Administração de Empresas do Departamento de Administração da PUC-Rio.

Orientador: Prof. Paulo César de Mendonça Motta

Rio de Janeiro

Abril de 2006 


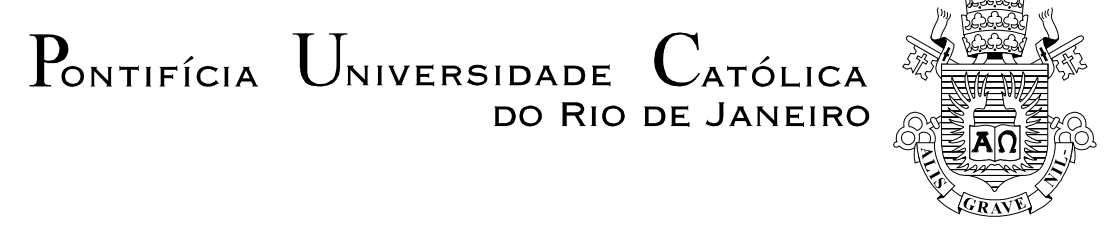

Jorge Luiz Correia de Araujo

\title{
A Criação de Valor Influenciando o Posicionamento \\ Estratégico - Estudo de Caso do Grupo RCH
}

\begin{abstract}
Dissertação apresentada como requisito parcial para obtenção do grau de Mestre pelo Programa de Pósgraduação em Administração de Empresas da PUC-Rio. Aprovada pela Comissão Examinadora abaixo assinada.
\end{abstract}

Prof. Paulo Cesar de Mendonça Motta
Orientador
Departamento de Administração - PUC-Rio

Prof. Jorge Alberto Zietlow Duro Flexcorp

Prof. Cecilia Lima de Queiros Mattoso ESPM

Prof. João Pontes Nogueira Vice-Decano de Pós-Graduação do CCS

Rio de Janeiro, 3 de abril de 2006 
Todos os direitos reservados. É proibida a reprodução total ou parcial do trabalho sem a autorização da universidade, do autor e do orientador.

\section{Jorge Luiz Correia de Araújo}

Graduou-se em Engenharia Química pela UFRJ (Universidade Federal do Rio de Janeiro) em 1984. Trabalhou em multinacionais como Texaco Chevron e Amitech Brasil sempre na área técnica comercial. Trabalha na RCH Automação Industrial desde 2004, na função de Gerente Comercial.

Ficha catalográfica

Araújo, Jorge Luiz Correia de

A Criação de Valor Influenciando o Posicionamento Estratégico - Estudo de Caso do Grupo RCH / Jorge Luiz Correia de Araújo ; orientador: Paulo César de Mendonça Motta. - Rio de Janeiro : PUC-Rio, Departamento de Administração, 2006.

100 f. ; $30 \mathrm{~cm}$

Dissertação (mestrado) - Pontifícia Universidade Católica do Rio de Janeiro, Departamento de Administração

Inclui bibliografia

1. Administração - Teses. 2. Modelo Delta. 3. Posicionamento estratégico. 4. Atributos. 5. Criação de valor. I. Motta, Paulo César de Mendonça. II. Pontifícia Universidade Católica do Rio de Janeiro. Departamento de Administração. III. Título.

CDD: 658 
Dedico esse sonho a três grandes mulheres, à minha esposa Liliane, por seu incondicional apoio e por nossa união, à minha filha Marcella pelo carinho e força e à minha mãe Nazaré, pelos ensinamentos preciosos dos valores morais e éticos.

O sonho é hoje realidade. 


\section{Agradecimentos}

A DEUS pela força indispensável nas horas difíceis, onde eu precisei lutar, ter discernimento e paciência para alcançar um grande sonho de minha vida.

Agradecimento especial ao meu orientador Professor Paulo César Motta pelo apoio e pelas valiosas idéias e reflexões que foram essenciais para a realização do trabalho.

A todos os meus ex - professores que transmitiram conhecimentos e participaram da minha formação.

Aos companheiros de turma que desde os tempos de Colégio Militar do RJ, da UFRJ e do IAG - PUC/ RJ, pela excelente convivência nesses 20 anos de estudo.

Ao meu pai que sempre está presente na minha vida.

A toda a minha família, em especial às minhas três irmãs (Ione, Jeane e Shirley) que me incentivaram e criaram um ambiente para que eu sempre estudasse.

Ao meu amigo-irmão Júlio que acreditou em mim e me ajudou na execução desse projeto de vida.

Aos meus sobrinhos que torcem pelo meu sucesso.

Aos meus sogros (Eliane e José Luiz) que vieram completar a minha família.

Aos meus cunhados (Lílian e Marco) pelo incentivo e ajuda na realização do trabalho.

Aos companheiros da empresa $\mathrm{RCH}$, em especial aos seus Diretores (Ferreira e Henrique) que me deram oportunidade para a realização desse trabalho.

Aos meus amigos que entenderam a minha ausência.

Aos entrevistados que contribuíram para a realização desse trabalho.

Ao povo brasileiro que me proporcionou ensino gratuito desde o ensino elementar até a graduação. 


\section{Resumo}

Araújo, Jorge Luiz Correia; Motta, Paulo César de Mendonça (Orientador). A Criação de Valor Influenciando o Posicionamento Estratégico Estudo de Caso do Grupo RCH. Rio de Janeiro, 2006. 100p. Dissertação de Mestrado - Departamento de Administração, Pontifícia Universidade Católica do Rio de Janeiro.

Com a constante evolução do mercado, o posicionamento estratégico nas organizações é uma preocupação recorrente de seus gestores; uma abordagem relevante é a utilização do modelo Delta proposto por Hax \& Wilde. O projeto Delta redefine a avaliação do mercado em três novas dimensões competitivas e justifica as forças econômicas mais complexas, integrando a estratégia à execução nos negócios. Foi construído um estudo de caso de acordo com a metodologia de Yin (2003), que utiliza dados coletados através de pesquisas documentais e análises qualitativas de entrevistas com a alta e média gerência assim como com os principais fornecedores e clientes do grupo RCH. Após a interpretação e análise dos dados, os resultados obtidos mostram os principais atributos valorizados pelos gestores, fornecedores e clientes da empresa, característicos do modelo Delta. O estudo identifica a importância da criação de valor e sua influência no reposicionamento estratégico do grupo RCH. Como resposta a essa proposição, foi construída uma figura denominada - Túnel do Tempo - que relaciona a linha do tempo com o modelo Delta e demonstra o deslocamento da dimensão estratégica do melhor produto para a dimensão estratégica de solução para o cliente desde a sua fundação até a data de hoje.

\section{Palavras-chave}

Modelo Delta; Posicionamento Estratégico; Atributos; Criação de Valor. 


\section{Abstract}

Araújo, Jorge Luiz Correia; Motta, Paulo César de Mendonça (Advisor). The Creation of Value Influencing the Strategical Positioning - Study of a Case of the Group RCH. Rio de Janeiro, 2006. 100p. Dissertation Departamento de Administração, Pontifícia Universidade Católica do Rio de Janeiro.

Due to the ongoing market evolution, the strategic positioning in most organizations is a recurrent concern among the managerial board; the use of the Delta Model proposed by Hax \& Wilde is a relevant approach. The Delta Project redefines the market evaluation in three new competitive dimensions and it accounts for more complex economic forces, linking business strategy to business execution. A case study has been built according to the Yin Methodology (2003), which uses collected data from documentary research and qualitative analysis of interviews done with RCH senior and middle management as well as with its main suppliers and customers. After data interpretation and analysis, results show the main attributes characteristic of the Delta Model of significant value to the Company's management, suppliers and customers. The study identifies the importance of value creation and its influence in the strategic repositioning of the group RCH. As an answer to this proposition, a figure named - Time Tunnel has been built. It relates the time line to the Delta Model and it shows the consequent change from strategic dimension of best product to strategic dimension of total customer solution since its foundation until present date.

\section{Keywords}

Delta Model; Strategic Positioning; Attributes; Value Creation. 


\section{Sumário}

1. Introdução 11

1.1. Definição do Problema 11

1.2. Objetivos Final e Intermediário 14

1.3. Delimitação do Estudo 14

1.4. Relevância do Estudo $\quad 15$

1.5. Estrutura da Dissertação 15

2. Revisão da Literatura $\quad 16$

2.1. A Criação de Valor e Inovação de Valor 16

2.2. A Inovação Estratégica 18

2.3. Posicionamento Estratégico 21

2.4. O Modelo Delta 25

2.4.1. O Triângulo 27

2.4.2. Os Processos Adaptativos $\quad 29$

2.4.3. Medições Agregadas $\quad 30$

2.4.4. Medições Granulares 31

2.4.5. Experimentação e Feedback 32

3. As Empresas do Grupo RCH 34

3.1. Histórico 34

4. Metodologia 37

4.1. Tipo de Pesquisa 37

4.2. Tratamento dos Dados 39

4.3. Limitações do Método 40

5. Análise de Tratamento dos Dados 41

5.1. Análise dos Dados 41

5.1.1. Atributos Percebidos 44

$\begin{array}{ll}\text { 5.2. Análises da Linha do Tempo } & 74\end{array}$

5.3. Considerações Finais

6. Discussão e Conclusão $\quad 89$

6.1. Discussão

6.2. Conclusão e Recomendações 91

$\begin{array}{ll}\text { Referências Bibliográficas } & 95\end{array}$

$\begin{array}{ll}\text { Anexo } & 98\end{array}$ 


\section{Lista de Tabelas}

Tabela 1 - Posicionamento Estratégico 24

Tabela 2 - Contribuições do Modelo Delta 26

Tabela 3 - Freqüência de Características - Modelo Delta 44

Tabela 4 - Princípios Básicos para Construção de Parceria 64 


\section{Lista de Figuras}

$\begin{array}{ll}\text { Figura } 1 \text { - Criação de Valor } & 18\end{array}$

Figura 2 - Triângulo Modelo Delta 27

Figura 3 - Modelo Delta - Um Diagrama de Estratégia Integrada 33

Figura 4 - Porcentagem de Vendas por Empresas ano 2005

Figura 5 - Triângulo Modelo Delta - Competição 36

Figura 6 - Principais Atributos Percebidos conforme Modelo Delta 73

Figura 7 - Gráfico dos Principais Atributos Percebidos 74

Figura 8 - Linha do Tempo $\quad 75$

Figura 9 - Linha do Tempo x Modelo Delta 93 